\title{
Atom counting in expanding ultracold clouds
}

\author{
Sibylle Braungardt, ${ }^{1}$ Mirta Rodríguez, ${ }^{2}$ Aditi Sen(De),${ }^{3}$ Ujjwal Sen, ${ }^{3}$ and Maciej Lewenstein ${ }^{1,4}$ \\ ${ }^{1}$ ICFO-Institut de Ciències Fotòniques, Mediterranean Technology Park, E-08860 Castelldefels (Barcelona), Spain \\ ${ }^{2}$ Instituto de Estructura de la Materia, CSIC, Serrano 121, E-28006 Madrid, Spain \\ ${ }^{3}$ Harish-Chandra Research Institute, Chhatnag Road, Jhunsi, Allahabad 211 019, India \\ ${ }^{4}$ Institució Catalana de Recerca i Estudis Avançats, Passeig Lluis Companys 23, E-08010 Barcelona, Spain
}

(Received 17 March 2011; published 21 October 2011)

\begin{abstract}
We study the counting statistics of ultracold bosonic atoms that are released from an optical lattice. We show that the counting probability distribution of the atoms collected at a detector located far away from the optical lattice can be used to characterize the initially trapped states. We consider trapped superfluid and insulating states with different occupation patterns. We analyze how the correlations between the modes that develop during the expansion in the gravitational field appear in the counting distribution and find that the ratio of the detector size with respect to the expanded wave function determines whether short-range or long-range correlations of the initial state are reflected in the counting statistics. We find that detectors which are large compared to the size of the expanded wave function distinguish insulating and superfluid phases irrespective of the occupation pattern. We show that using detectors that are small compared to the size of the expanded wave function, occupation patterns in insulating and supersolid states can be distinguished. Finally, we show how the magnetic phase patterns are dramatically reflected in the number distribution.
\end{abstract}

DOI: 10.1103/PhysRevA.84.043635

PACS number(s): 67.85.-d, 37.10.Jk, 05.30.Jp

\section{INTRODUCTION}

Experiments with ultracold particles trapped in optical lattices aim toward the engineering of exotic many-body quantum states [1]. Recently, trapping and cooling of dipolar gases have attracted much attention [2]. The dipole moments induce long-range interactions between the particles, and new phases appear [3]. In the strongly correlated regime, it has been shown that there are many quasidegenerate metastable insulating states with defined occupation patterns [4-7]. These metastable states could be used for the storage and processing of quantum information analogously to classical neural networks, where the information is robustly encoded in the distributed stable states of a complex system [8,9]. Another way to induce long-range interactions and thus control the lattice occupation patterns is via coupling to an external cavity mode. This has just recently been achieved experimentally and a checkerboard to a supersolid transition has been observed [10]. Additionally, the creation of artificial gauge phases in neutral atoms is an active area of research [11]. Dressing the lattice with a high-frequency acceleration [12] results in an effective tunneling rate between lattice sites which can be used to create states with staggered phase patterns which mimic magnetic Néel order [13].

The detection of exotic strongly correlated phases requires novel experimental techniques that give access to high-order correlation functions. Proposals for detection techniques typically make use of shot-noise measurements [14] or atom-light interfaces [15]. Also, the counting statistics of atoms have been suggested as a technique able to distinguish strongly correlated [16-19] and fermionic [20,21] Hamiltonians, as well as systems of interacting Rydberg atoms [22]. The detection of atoms trapped in the optical lattice with single-site resolution has become experimentally available only recently [23-26]. Most counting experiments are performed after switching off the trapping potential and letting the atoms propagate in the gravitational field. The counting statistics of $\mathrm{Rb}$ atoms falling within a high-finesse cavity has been reported in Ref. [27]. Also, fermionic and bosonic counting probability distributions have been measured for metastable Helium atoms falling onto a microchannel plate $[28,29]$.

The theoretical analysis of the counting process has so far mainly been considered for atoms trapped in the lattice. Propagation in the gravitational field mixes the initial modes of the atoms such that the counting statistics in the lattice and after propagation are not expected to be the same. In this paper, we study the role of expansion in the counting process. We show that the mixing of the initial modes during the expansion becomes evident in the counting distribution when the detector is small compared to the size of the expanded wave function. We illustrate the effect by analyzing the counting statistics for bosons after time-of-flight expansion from the lattice. We consider initial states with different occupation patterns in the insulating regime and states with different density and phase distributions in the superfluid regime. We calculate both the counting probabilities at a single detector and the joint probabilities at two detectors as a function of the horizontal distance between them. We show that superfluid (SF) and Mott-insulator (MI) phases can be distinguished by their counting statistics. We further show that a suitable choice of the detector geometry, or equivalently the distance to the detector, allows for the characterization of different occupation patterns in the insulating regime and different supersolid states. Moreover, we find that phase patterns are also reflected in the counting statistics.

The paper is organized as follows. In Sec. II we review the propagation of the atomic wave functions and the atom counting formalism. In Sec. III we analyze the intensity of particles arriving at the detector, which consists of autocorrelation terms and cross correlations between the different expanded modes. Depending on the distance and size of the detector, the ratio between the autocorrelations and the cross-correlation terms changes. In Sec. IV we obtain closed expressions for the counting distributions for expanded superfluid and 
insulating bosonic states. We consider the counting statistics when using one detector and the joint counting distribution at two detectors. In Sec. V, we collect our results and compare the counting distributions of SF and MI states and the counting distributions for insulating and supersolid states with different occupation patterns as well as magnetic phase patterns.

\section{DESCRIPTION OF THE SYSTEM}

We consider neutral bosonic atoms trapped in an optical lattice. The system can be described using the Bose-Hubbard model [30], which includes the hopping of the particles between neighboring sites and the on-site two-body interactions. At zero temperature, there are two limiting states in the phase diagram. On the one hand, when the hopping term dominates, SF states with long-range correlations appear. Fast oscillatory potentials can be used to dress the lattice [12] and produce an effective tunneling rate. By controlling the parameters of the driving, one can obtain negative effective tunneling rates which induce staggered phase patterns which mimic magnetic Néel order [13]. On the other hand, the opposite limit appears when the interparticle interactions are dominant, and the ground states are MI states which lack any long-range correlations. If, additionally, the interactions between particles are long range $[3,10]$, the limiting ground states of the system are MI and SF states with inhomogeneous occupation patterns.

The field operator $\Psi(\mathbf{r}, t)$ of the many-body system can be expanded into the $N$ modes $a_{i}$

$$
\Psi(\mathbf{r}, t)=\sum_{i} \phi_{i}(\mathbf{r}, t) a_{i} .
$$

For atoms trapped in an optical lattice, $a_{i}$ describes the destruction of a particle on site $i$ with a single-particle wave function $\phi_{i}$. The corresponding initial wave functions are Wannier functions which are Gaussian functions centered at $\mathbf{r}_{i}$

$$
\phi_{i}(\mathbf{r}, t=0)=\frac{1}{\left(\pi \omega^{2}\right)^{3 / 4}} e^{-\left(\mathbf{r}-\mathbf{r}_{i}\right)^{2} / 2 \omega^{2}},
$$

where the width $\omega$ is chosen such that the initial wave functions at different sites $i$ have a negligible overlap.

The atoms are released from the optical lattice and expand in the gravitational field. At finite $t$, we can apply the singleparticle expansion

$$
\phi_{i}(\mathbf{r}, t)=\int d \mathbf{r}^{\prime} K\left(\mathbf{r}, \mathbf{r}^{\prime}, t\right) \phi_{i}\left(\mathbf{r}^{\prime}, 0\right)
$$

where the propagator for the free expansion in the gravitational field reads [31]

$$
K\left(\mathbf{r}, \mathbf{r}^{\prime}, t\right)=\left(\frac{m}{2 \pi i \hbar t}\right)^{3 / 2} e^{\frac{i m\left(\mathbf{r}-\mathbf{r}^{\prime}\right)^{2}}{2 \hbar t}-\frac{i m g t\left(z+z^{\prime}\right)}{2 \hbar}-\frac{i m^{2} g^{2} t^{3}}{24 m \hbar}} .
$$

The full propagated wave function is then written as

$$
\phi_{i}(\mathbf{r}, t)=\frac{e^{-\frac{i m^{2} g^{2} t^{3}}{24 m \hbar}}}{\pi^{3 / 4}\left(i \omega_{t}+\omega\right)^{3 / 2}} e^{-\frac{\left(\mathbf{r}-\mathbf{r}_{i}\right)^{2}}{2\left(\omega_{t}^{2}+\omega^{2}\right)}} e^{-i \frac{\left(\mathbf{r}-\mathbf{r}_{i}\right)^{2} \omega_{t}}{2 \omega\left(\omega_{t}^{2}+\omega^{2}\right)}},
$$

where and $\mathbf{r}_{t}=\mathbf{r}+\mathbf{z}_{t}$, with $\mathbf{z}_{t}=\left(0,0, g t^{2} / 2\right)$ and we have used that $\left|\mathbf{r}_{t}-\mathbf{r}_{i}\right| \gg \omega$. In the limit of $\omega_{t} \gg \omega$, the expanded wave function is, up to a phase factor, a Gaussian function centered around $\mathbf{z}_{t}$ with a width $\omega_{t}=\hbar t /(m \omega)$.

\section{A. Atom counting}

We describe a counting process in which the probability $p(m)$ of counting $m$ particles within a time interval $\tau$ is measured at a detector located at a distance $z_{0}$ from the lattice. The probability of detecting $m$ particles can be expressed as $[32,33]$

$$
p(m)=\left.\frac{(-1)^{m}}{m !} \frac{d^{m}}{d \lambda^{m}} \mathcal{Q}\right|_{\lambda=1},
$$

where the generating function $\mathcal{Q}(\lambda)$ is given by the expectation value of a normally ordered exponential of the intensity $\mathcal{I}$,

$$
\mathcal{Q}(\lambda)=\operatorname{Tr}\left(\rho: e^{-\lambda \mathcal{I}}:\right) .
$$

For photons, the intensity is proportional to an integral over the product of the negative-frequency part and the positivefrequency part of the field. The normal ordering : . . . : reflects the detection mechanism, in which the photons are absorbed at the detector, typically a photo multiplier or an avalanche photodiode. For the detection of atoms using microchannel plates, the detection process can be treated in an analogous way.

Since typically not all the particles are counted, the intensity depends on the efficiency $\epsilon$ of the detector and the detection time $\tau$. When the dynamics of the measurement are fast in comparison to the dynamics of the system, the intensity is proportional to the factor $\kappa \equiv 1-e^{-\epsilon \tau}$. For typical experimental situations, the dynamics of the system are determined by the expansion of the atomic cloud in the gravitational field, given by $\omega_{t}$ and the intensity can be described by the integral over the detector volume $\Omega$ of the positive-frequency and negative-frequency parts of the quantum fields describing the particles to be counted, multiplied by the efficiency factor $\kappa[34]$,

$$
\mathcal{I}=\kappa \int_{\Omega} d \mathbf{r} \Psi^{\dagger}\left(\mathbf{r}, t_{d}\right) \Psi\left(\mathbf{r}, t_{d}\right),
$$

where $t_{d}$ denotes the time at which the instantaneous measurement is performed.

The formalism described above is easily generalized to the case of detection with multiple detectors [35]. For detection with $M$ detectors, the generating function reads

$$
\mathcal{Q}_{M}\left(\lambda_{1}, \lambda_{2}, \ldots, \lambda_{M}\right)=\operatorname{Tr}\left(\rho: e^{-\sum_{i} \lambda_{i} \mathcal{I}_{i}}:\right),
$$

where the single detector intensity $\mathcal{I}_{i}$ for each of the detectors is given by Eq. (8). For a configuration with two detectors, the joint probability distribution of counting $m$ atoms at detector 1 and $n$ atoms at detector 2 is given by

$$
p(m, n)=\left.\frac{(-1)^{m+n}}{m ! n !} \frac{d^{m+n}}{d \lambda_{1}^{m} d \lambda_{2}^{n}} \mathcal{Q}_{2}\right|_{\lambda_{1}=1, \lambda_{2}=1} .
$$

We study the correlations $\operatorname{corr}(m, n)$ between the counting events detected at each detector by observing the ratio between the covariance and the single detector variances,

$$
\operatorname{corr}(m, n)=\frac{\operatorname{cov}(m, n)}{\sigma_{m}^{2} \sigma_{n}^{2}},
$$

where $\operatorname{cov}(m, n)=\sum_{m, n} m n p(m, n)-\bar{m} \bar{n}, \bar{m}$ denotes the mean and $\sigma_{m}^{2}$ the variance of $p(m)$. 


\section{DETECTION OF EXPANDING ATOMS}

\section{A. Correlations between the expanding modes}

Let us now discuss the counting process for the detection of atoms expanding in the gravitational field. We consider a cubic detector located at a distance $z_{0}$ from the lattice center with edge lengths $\Delta_{x}, \Delta_{y}, \Delta_{z}$. For simplicity, all through this paper we consider $t_{d}=\sqrt{2 z_{0} / g}$ which is the time when the center of the cloud arrives at the detector. The intensity $\mathcal{I}$ of atoms registered at the detector defined in Eq. (8) is thus determined by the expanded field operator of the atoms at the time $t_{d}$ of detection, $\Psi\left(z_{0}, t_{d}\right)$. Using Eqs. (1) and (8), the intensity $\mathcal{I}$ takes the form

$$
\mathcal{I}=\sum_{i j} A_{i j} a_{i}^{\dagger} a_{j}
$$

where

$$
A_{i j}\left(z_{0}, \Omega, \kappa\right)=\kappa \int_{\Omega} d \mathbf{r} \phi_{i}^{*}\left(z_{0}, t_{d}\right) \phi_{j}\left(z_{0}, t_{d}\right) .
$$

The elements of the correlation matrix $A_{i j}$ defined in Eq. (13) describe the interference and autocorrelation terms between different modes registered at the detector. The diagonal terms represent the on-site correlations, whereas the off-diagonal terms represent the cross correlations between single-particle modes initially located at different sites with distance $\mathbf{d}=$ $\mathbf{r}_{j}-\mathbf{r}_{i}$.

Before studying the full counting distribution, let us consider the correlations given by the matrix elements $A_{i j}$. Using Eq. (5) and assuming $\omega_{t_{d}} \gg \omega$, the autocorrelation elements are given by

$$
A_{i i}=\kappa \int_{\Omega} d \mathbf{r} \frac{1}{\pi^{3 / 2} \omega_{t_{d}}^{3}} e^{-\frac{\left(\mathbf{r}-\mathbf{r}_{i}\right)^{2}}{\omega_{t_{d}}^{2}}} .
$$

For expanded wave functions at $\mathbf{r} \gg \mathbf{r}_{i}$, the autocorrelations become all equal $A_{i i} \simeq A_{D}$ and independent of the original lattice site $i$,

$$
A_{D}=\kappa \prod_{l=x, y, z} \operatorname{Erf}\left(\frac{\Delta_{l}}{\omega_{t_{d}}}\right) .
$$

The cross correlations are given by

$$
A_{i j}=\kappa \int_{\Omega} d \mathbf{r} \frac{1}{\pi^{3 / 2} \omega_{t}^{3}} e^{-\frac{\left(\mathbf{r}-\mathbf{r}_{i}\right)^{2}}{2 \omega_{t}^{2}}} e^{-\frac{\left(\mathbf{r}-\mathbf{r}_{j}\right)^{2}}{2 \omega_{t}^{2}}} e^{-i \frac{\mathbf{r}\left(\mathbf{r}_{i}-\mathbf{r}_{j}\right)}{\omega \omega_{t}}} .
$$

If, again, we assume far detection, $A_{i j}$ depends only on $\mathbf{d}=$ $\mathbf{r}_{j}-\mathbf{r}_{i}$ and thus

$$
\begin{aligned}
A_{i j}= & \kappa \prod_{l=x, y, z} \frac{i e^{-\left(\frac{d_{l}}{4 \omega}\right)^{2}}}{2}\left[\operatorname{Erfi}\left(\frac{d_{l}}{2 \omega}+\frac{i \Delta_{l}}{\omega_{t_{d}}}\right)\right. \\
& \left.-\operatorname{Erfi}\left(\frac{d_{l}}{2 \omega}-\frac{i \Delta_{l}}{\omega_{t_{d}}}\right)\right],
\end{aligned}
$$

where $a_{0}$ is the lattice constant and $d_{l}$ is the $l$ component of the distance between the two sites, where $l=x, y, z$.

Both the cross correlations and the autocorrelations depend crucially on $\Delta_{l} / \omega_{t_{d}}$ which can be manipulated by changing the distance to the detector $z_{0}$ or by changing the detector size. In Fig. 1, we show the autocorrelations and the crosscorrelation terms as a function of the size of the detector. We
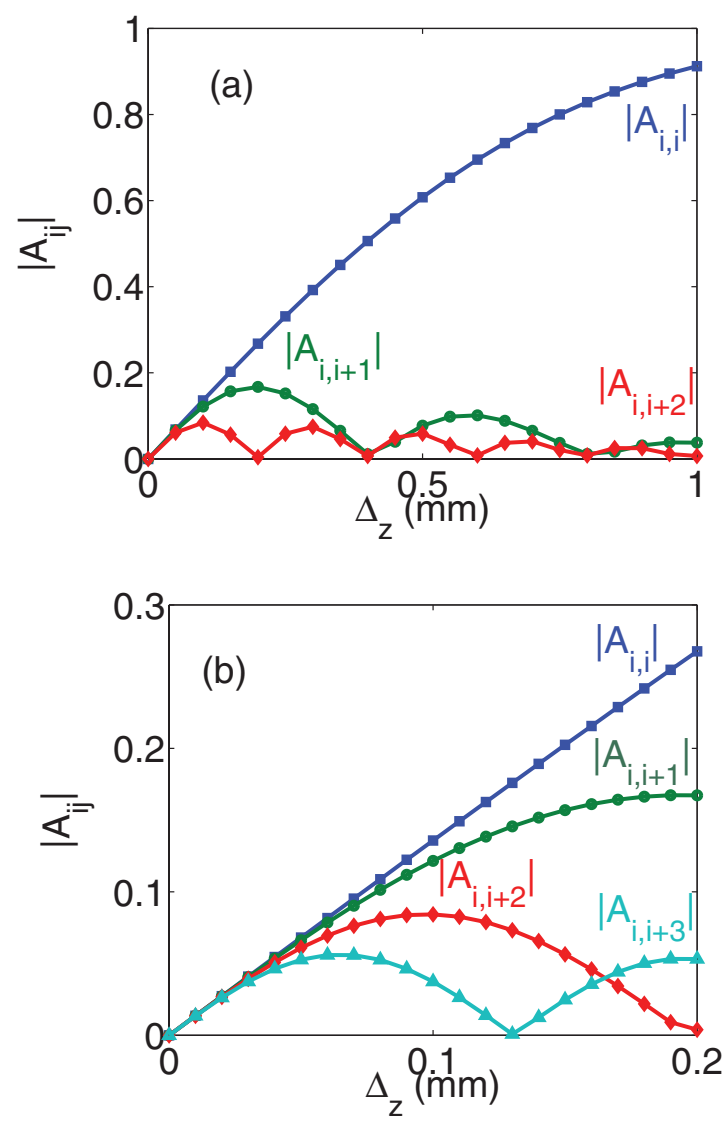

FIG. 1. (Color online) The ratio between the autocorrelations and cross-correlation terms that contribute to the counting distribution depend on the size of the detector. For a 1D system, we plot the following: (a) wide detector limit $\Delta_{z} \simeq \omega_{t_{d}}$; (b) narrow detector limit $\Delta_{z} \ll \omega_{t_{d}}$. We plot $\left|A_{i i}\right|$ (blue squares) $\left|A_{i, i+1}\right|$ (green circles), $\left|A_{i, i+2}\right|$ (red diamonds), and $\left|A_{i, i+3}\right|$ (light blue triangles). Parameters used: $z_{0}=1 \mathrm{~cm}, \Delta_{x}=\Delta_{y}=1 \mathrm{~cm}$, and $\kappa=1, a_{0}=266 \mathrm{~nm}$.

consider a one-dimensional array in the $z$ direction and plot the correlations at the location of the detector $\left(0,0, z_{0}\right)$. We consider a fixed detector size in the $x y$ plane, $\Delta=\Delta_{x}=\Delta_{y}$, and vary its width $\Delta_{z}$. For $z_{0}=1 \mathrm{~cm}$, the size of the expanded single-particle wave function at the detector is $\omega_{t_{d}}=0.8 \mathrm{~mm}$. Depending on the size of the detector, the whole cloud or a fraction of it is registered. In the limit of large detectors $\Delta_{z} / \omega_{t} \rightarrow \infty$ we expect from Eq. (15) and (17) that $A_{D}$ approaches a constant value $\kappa$ while $A_{i j} \rightarrow 0$ for $i \neq j$. In Fig. 1(a), we show that for a 1D system with the given parameters, the interference terms are negligible for detectors of size $\Delta_{z}>0.2 \mathrm{~mm}$. This is easily understood from Eq. (14), as the autocorrelations are given by an integral over the detector volume around the center of a Gaussian function. For detectors that are large compared to the size of the cloud, the on-site correlations approach unity. In contrast, the interference terms Eq. (16) are given by the integral over a Gaussian function multiplied by a highly oscillating phase such that they approach zero as the size of the detector increases. Figure 1(b) shows that for small detector sizes, the interference terms for sites that are close to each other are of the same order as the on-site correlations. Note that due to the damping in 
Eq. (17), cross correlations for sites with large distances become zero for any detector size.

\section{B. Detection of the correlations}

We have seen in the previous section that when the detector is larger than the size of the cloud, only autocorrelations between the modes are registered at the detector. We see in Fig. 1(a) that the cross correlations disappear $A_{i j} \simeq 0$ for large distances between the sites $i$ and $j$, while the autocorrelations approach $A_{i i} \simeq \kappa$. Note that on-site detection is a particular limit of this case. We will show in the next section that the detection of the autocorrelations between different modes is sufficient to distinguish the long-range spatial correlations in the probed system. In particular, we show that in the limit of large detectors, MI states can be distinguished from SF states. On the contrary, as the autocorrelation terms for different sites are equal, distinguishing states with different occupation patterns cannot be achieved in this limit.

In order to characterize the occupation or phase pattern in a state, we need to use a detector which is small compared to the expanded function. In such a case not only autocorrelation terms but also cross correlations between the modes are registered at the detector. In particular, in order to distinguish patterns that differ at a distance $\mathbf{d}=\mathbf{r}_{j}-\mathbf{r}_{i}$, the optimal situation to achieve this would be that the registered cross correlations and autocorrelations are of the same order $\left|A_{i j}\right| \simeq$ $\left|A_{i i}\right|$. This can be achieved by choosing the proper detector size. For example, in a 1D system, if one wants to distinguish occupation or phase patterns with differences in the nearest neighbors, a choice of $\Delta_{z}=0.2 \mathrm{~mm}$ for the parameters used in Fig. 1 yields $\left|A_{i j}\right| \simeq\left|A_{i i}\right|$ for $j=i+1$ and $\left|A_{i j}\right| \rightarrow 0$ for $j>i+1$. We will show in Sec. $\mathrm{V}$ that in the limit of small detectors, different occupation and phase patterns are reflected in the counting distribution.

Note that the key parameter is the ratio between the expanded wave function $\omega_{t_{d}}$ and the detector size and one can thus either change the detector size $\Delta_{z}$ or the distance to the detector $z_{0}$.

\section{ATOM COUNTING STATISTICS}

Let us now consider the counting distributions measured at the detector after the expansion for different initial states of the system of atoms trapped in the lattice.

\section{A. Superfluid phases}

First, let us focus on SF states, ground states of the Bose-Hubbard model for very shallow lattices. We derive the counting distribution using the Gutzwiller ansatz [36] for the wave function which assumes that it is a product of on-site coherent states. The initial state of the atoms in the lattice with $N$ sites then reads:

$$
|\psi\rangle=\prod_{i}^{N}\left|\alpha_{i}\right\rangle_{i}
$$

where $\left|\alpha_{i}\right\rangle_{i}$ is the coherent state on site $i$,

$$
\left|\alpha_{i}\right\rangle_{i}=e^{-\left|\alpha_{i}\right|^{2} / 2} \sum_{n=0}^{\infty} \frac{\alpha_{i}^{n}}{\sqrt{n !}}|n\rangle_{i}
$$

and $|n\rangle_{i}=\frac{1}{\sqrt{n !}}\left(a_{i}^{\dagger}\right)^{n}|0\rangle$ is a Fock state with $n$ particles. Note that $|\psi\rangle$ is an eigenstate of the annihilation operator $\Psi(\mathbf{r}, t)$ of the expanded atoms,

$$
\Psi(\mathbf{r}, t)|\psi\rangle=\sum_{i} \phi_{i}(\mathbf{r}, t) \alpha_{i}|\psi\rangle,
$$

where $\phi_{i}$ is given by Eq. (2). The state $|\psi\rangle$ is thus an eigenstate of the expanded field operator $\Psi(\mathbf{r}, t)$ and we can write the generating function as $\mathcal{Q}(\lambda)=e^{-\lambda \sum_{i j} \alpha_{i}^{*} \alpha_{j} A_{i j}}$. Using Eq. (6) the counting distribution $p(m)$ reads

$$
p(m)=\frac{\left(\sum_{i j} \alpha_{i}^{*} \alpha_{j} A_{i j}\right)^{m}}{m !} e^{-\sum_{i j} \alpha_{i}^{*} \alpha_{j} A_{i j}},
$$

where $A_{i j}$ is given by Eq. (13). This corresponds to a Poissonian distribution with mean (and thus also variance) $\bar{m}=\sigma^{2}=\sum_{i j} \alpha_{i}^{*} \alpha_{j} A_{i j}$.

In the limit of big detectors where the diagonal elements of the matrix $A_{i j}$ are much bigger than the off-diagonal elements, all $A_{i i}=A_{D}$. In such case the number distribution reads

$$
p(m)=\frac{\left(\sum_{i}\left|\alpha_{i}\right|^{2} A_{D}\right)^{m}}{m !} e^{-\sum_{i}\left|\alpha_{i}\right|^{2} A_{D}},
$$

which does not distinguish different occupation patterns with the same number of particles.

If one wants to characterize the occupation pattern, one needs to choose smaller detector sizes such that some cross correlations $A_{i j}$ are nonzero. In Sec. V we show that in the limit where the nearest-neighbor cross-correlation contribution is of the same order than the autocorrelations one can use the number distribution to characterize supersolid states.

\section{B. Mott-insulating phases}

Let us now consider initial states in the Mott-insulating regime. We first study a Mott-insulator state with one particle per site, $|\psi\rangle=|11 \ldots 11\rangle$. In this case, the generating function Eq. (7) reads

$$
\begin{aligned}
\mathcal{Q}(\lambda) & =\left\langle 11 \ldots 11\left|: e^{-\lambda \kappa \int_{\Omega} d \mathbf{r} \Psi^{\dagger}\left(\mathbf{r}, t_{d}\right) \Psi\left(\mathbf{r}, t_{d}\right)}:\right| 11 \ldots 11\right\rangle \\
& =1-\lambda \sum_{i} A_{i i}+\lambda^{2} \sum_{i<j}\left(A_{i i} A_{j j}+\left|A_{i j}\right|^{2}\right)-\ldots
\end{aligned}
$$

We can rewrite Eq. (23) using the minors of the matrix $A$,

$$
\mathcal{Q}(\lambda)=1+\sum_{k=1}^{N}(-1)^{k} \lambda^{k} \mathrm{M}_{+}(A, k),
$$

where $\mathbf{M}_{+}(A, m)$ denotes the permanent $\operatorname{perm}(A)=$ $\sum_{\sigma \in S_{n}} \Pi_{i=1}^{n} A_{i, \sigma(i)}$ of the corner blocks of size $m$ of the matrix $A$. Note that $\mathrm{M}_{+}(A, k)$ is closely related to the principal minors of the matrix, which are defined as the determinant of the respective block matrices. The counting distribution $p(m)$ can then be calculated using Eqs. (6) and (24). 
In an experimental situation where the detector is located far away from the lattice and much bigger than the cloud, the generating function $\mathcal{Q}$ in Eq. (23) reduces to

$$
\mathcal{Q}(\lambda)=\sum_{k=0}^{N}\left(\begin{array}{l}
N \\
k
\end{array}\right)\left(-\lambda A_{D}\right)^{k}=\left(1-A_{D} \lambda\right)^{N},
$$

where $A_{D}$ denotes any of the (equal) diagonal elements. The counting distribution $p(m)$ is then given by

$$
p(m)=\left(\begin{array}{l}
N \\
m
\end{array}\right) A_{D}^{m}\left(1-A_{D}\right)^{N-m} .
$$

This corresponds to the distribution of a Fock state. The mean $\bar{m}$ and variance $\sigma^{2}$ of the distribution are given by

$$
\bar{m}=N A_{D}, \quad \sigma^{2}=N A_{D}\left(1-A_{D}\right) .
$$

Let us now consider the different occupation patterns that arise in the strongly correlated regime. In particular, we focus on such states where at most one particle occupies each site. The generating function is then calculated by Eq. (24), with a correlation matrix $A^{\prime}$, composed of the elements of the correlation matrix $A$ in Eq. (13) multiplied by the occupation numbers $n_{i}$ and $n_{j}$ of the involved sites,

$$
A^{\prime}=n_{i} n_{j} A_{i j} .
$$

Note that in the limit when the detector is large only the autocorrelations are nonzero and the number probability distributions for all the Mott-insulating states discussed in this section are same as in Eq. (26) with $N$ equal to the number of particles.

\section{Counting at two detectors}

In this section, we consider the detection of MI and SF phases using two detectors and analyzing the correlations between the counting events. For detectors that are located symmetrically with respect to the origin in the $x y$ plane, and in an experimental situation such that only the autocorrelation terms are non-negligible, we obtain joint probability distributions for MI and SF states which are independent of the occupation patterns.

For the MI state, the joint counting distribution $p(m, n)$ of counting $m$ particles at one detector and $n$ particles at the other is given by Eq. (10), where the generating function for two detectors is given by

$$
\mathcal{Q}_{2}=\sum_{k=1}^{N}(-1)^{k} M^{+}\left(\lambda_{1} A^{(1)}+\lambda_{2} A^{(2)}, k\right) .
$$

For large detectors that are located symmetrically with respect to the origin in the $x y$ plane, the diagonal elements $A_{D}$ are all equal, $A_{D}^{(1)}=A_{D}^{(2)}=A_{D}$. The generating function thus simplifies to

$$
\begin{aligned}
\mathcal{Q}_{2}\left(\lambda_{1}, \lambda_{2}\right) & =\sum_{k=0}^{N}\left(\begin{array}{l}
N \\
k
\end{array}\right)\left(-A_{D}\right)^{k}\left(\lambda_{1}+\lambda_{2}\right)^{k} \\
& =\left[1-A_{D}\left(\lambda_{1}+\lambda_{2}\right)\right]^{N},
\end{aligned}
$$

and the counting distribution is given by

$$
\begin{aligned}
p(m, n)= & (-1)^{n+m}\left(1-2 A_{D}\right)^{N-m-n} \\
& \times\left(-A_{D}\right)^{m+n} \frac{N !}{m ! n !(N-m-n) !} .
\end{aligned}
$$

For the SF phases, the joint counting distribution $p_{\mathrm{SF}}(m, n)$ is the product of the two single detector distributions $p_{1}(m)$ and $p_{2}(n)$ given by Eq. (21). The counting events at the two detectors are thus not correlated.

\section{RESULTS}

\section{A. Counting distribution of Mott-insulating and superfluid phases}

We consider the counting distributions of a SF and a MI state of bosons with the same average number of particles released from a three-dimensional optical lattice. We assume the limit of a large detector, where the counting distribution is determined by the on-site correlation terms. In Fig. 2, we plot the counting distributions for an SF and an MI state at different distances between the detector and the lattice. With increasing distance from the detector, a smaller fraction of the expanded wave function is registered. The difference between the MI and the SF becomes less visible, and the mean of the counting distribution decreases. In Fig. 3, we plot the mean and the variance of the counting distributions, both normalized by
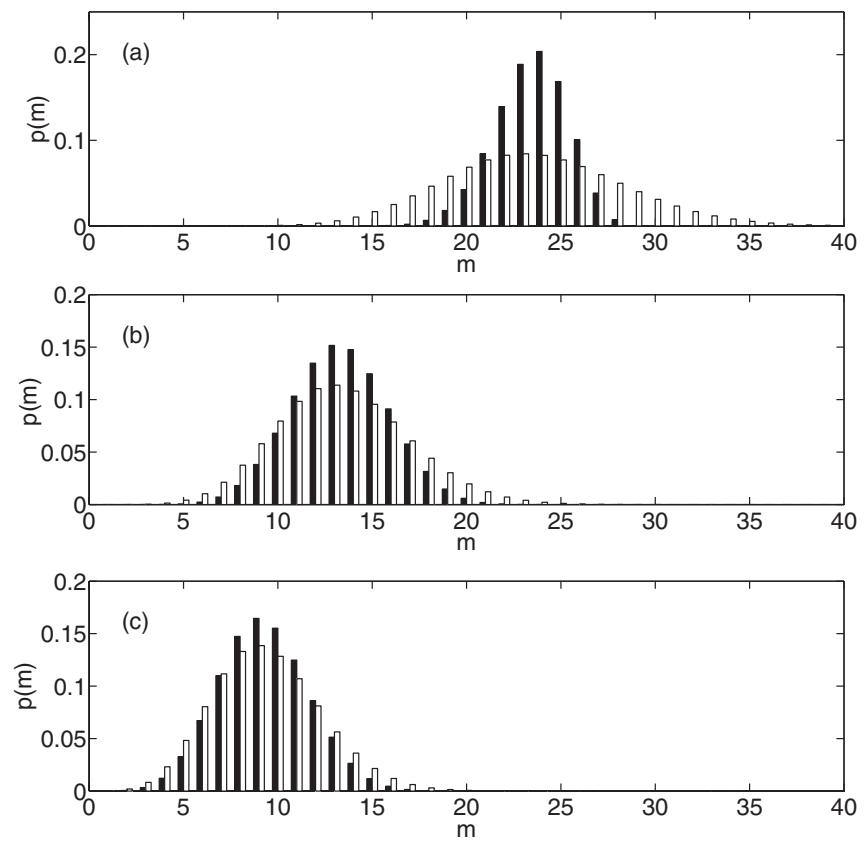

FIG. 2. MI vs. SF as a function of distance from detector. Probability distribution for MI (black bars) and superfluid (white bars) states in a $3 \times 3 \times 3$ lattice. The counting distribution of the SF state is Poissonian, whereas the counting distribution of the MI state is sub-Poissonian. For increasing distance between the detector, the difference between the states is no longer visible. $\Delta_{x}=\Delta_{y}=$ $2 \mathrm{~mm} ; \Delta_{z}=2 \mathrm{~cm} ; \kappa=1$. (a) $z_{0}=1 \mathrm{~cm} ;$ (b) $z_{0}=3 \mathrm{~cm}$; (c) $z_{0}=5 \mathrm{~cm}$. 


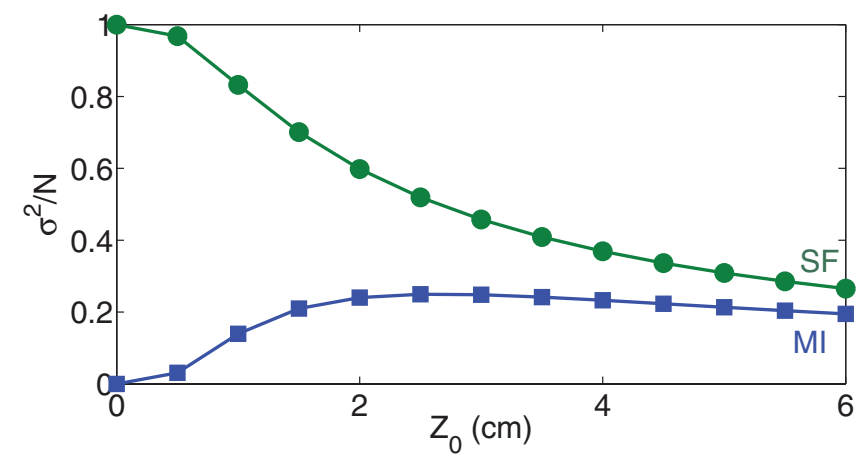

FIG. 3. (Color online) Variance $\sigma^{2} / N$ of the counting distribution for a MI (blue squares) and SF (green circles) state in a $3 \times 3 \times 3$ lattice with respect to the distance from the detector $z_{0} . \Delta_{x}=\Delta_{y}=2$ $\mathrm{mm} ; \Delta_{z}=2 \mathrm{~cm} ; \kappa=1$.

dividing by $N$, for the superfluid and the Mott-insulator state for a detector with fixed size at different distances $z_{0}$ from the lattice.

Let us now consider two detectors of the same size that are placed symmetrically at a distance $\mathbf{x}_{1}=\left(x_{d}, 0, z_{0}\right)$ and $\mathbf{x}_{2}=\left(-x_{d}, 0, z_{0}\right)$ from the lattice center. In the limit of large detectors, we study the joint counting distribution of the SF and MI states for different distances between the detectors. Figure 4 shows the counting distributions for two overlapping detectors (left column) and for two detectors separated by $2 x_{d}=1 \mathrm{~cm}$ (right column). For the SF state, shown in the lower row in Figs 4, the joint counting distribution is a Gaussian function for both cases. This is expected, as the joint counting distribution Eq. (21) is a product of the single detector counting distributions. This is analogous to the detection of coherent states of light. For the MI state shown in the upper row in Fig. 4, we observe a squeezed distribution, indicating the correlations of the atoms counted at the two detectors. As
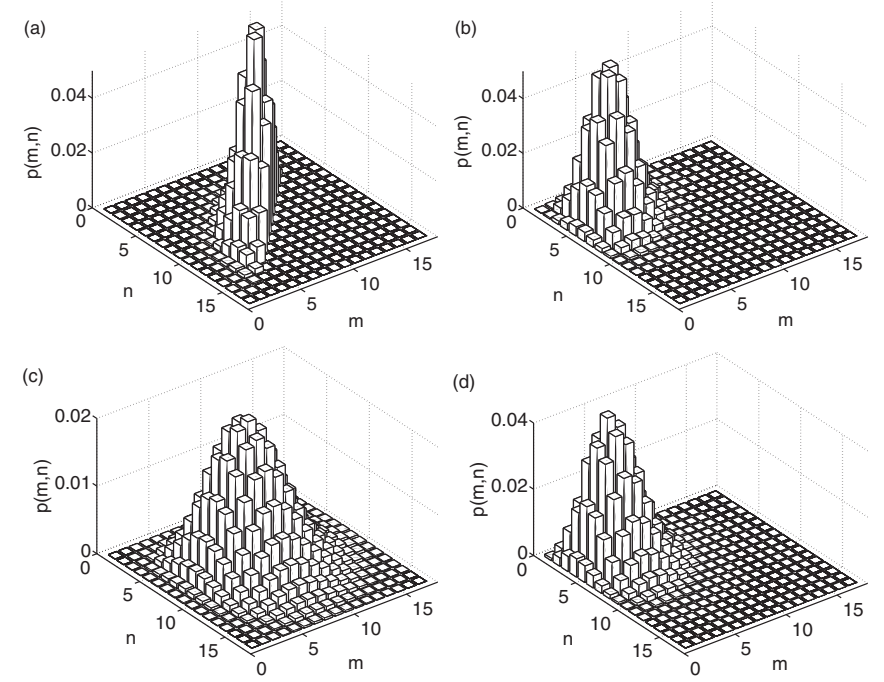

FIG. 4. Joint probability distribution of an expanded MI (upper row) and a SF (lower row) in a $4 \times 4$ lattice in the $x y$ plane with two symmetrically placed detectors. In (a) and (c) $x_{d}=0$. In (b) and (d) $x_{d}=1 \mathrm{~cm}$. Parameters used: $z_{0}=1 \mathrm{~cm} ; \Delta_{z}=2 \mathrm{~mm} ; \Delta_{x}=\Delta_{y}=$ $2 \mathrm{~cm} ; \kappa=0.5$.

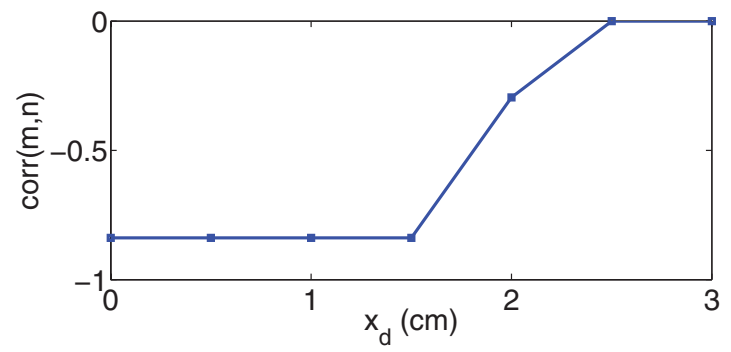

FIG. 5. (Color online) Correlations of the joint probability distribution for an expanded MI state in a $4 \times 4$ lattice in the $x y$ plane registered at two symmetrically placed detectors. For short distances an antibunching of the particles is observed. As the distance between the detectors increases, the counting events at the two detectors are no longer correlated. Parameters used: $z_{0}=1 \mathrm{~cm} ; \Delta_{z}=2 \mathrm{~mm}$; $\Delta_{x}=\Delta_{y}=2 \mathrm{~cm} ; \kappa=0.5$.

the distance between the detectors increases, the squeezing of the distribution is less pronounced. The correlations between the counting events at the two detectors can be seen more clearly when looking at the correlation function Eq. (11). For the superfluid state, there is no difference between the joint counting distribution and the product of the single particle distributions. For the Mott state, we study the correlations for varying distance between the two detectors $x_{d}$. In Fig. 5, we show how the correlations decrease when increasing the distance between detectors $x_{d}$. For detectors that are close together, an antibunching of the MI state is observed. The distance $x_{d}$ denotes the distance between the center of the two detectors. For $x_{d}=0$, the detectors fully overlap, and for $x_{d}>\Delta$ the detectors are completely separated. Timeof-flight expansion images readily distinguish between the MI and the SF regime [30]. Particle counting complements the characterization of the states by giving access to the full statistics.

All the results in this section are independent of the occupation pattern and are thus valid for any SF or MI state with up to unit filling. In order to characterize occupation patterns and short-range correlations in the initial states, one needs to use detectors of smaller sizes as we show in the next sections.

\section{B. Counting statistics of insulating states with different occupation patterns}

Let us now focus on the characterization of insulating states with different occupation patterns by particle counting. As discussed above, in order to detect the different patterns, the cross correlations have to be of the order of the autocorrelations. This is clear as away from the lattice, all the on-site correlation terms become equal. Let us discuss the example of a checkerboard state, where every second site is occupied, and a state with stripes, where every second line is occupied. For the striped state, the leading cross-correlation terms Eq. (16) are the ones that correspond to the nearest neighbors. For the checkerboard state, where neighboring sites are not occupied, the leading terms are the ones that correspond to diagonally adjacent sites. In order to distinguish the different patterns, it is, thus, essential that these two leading cross-correlation terms differ sufficiently and, at the same time, are comparable to the 


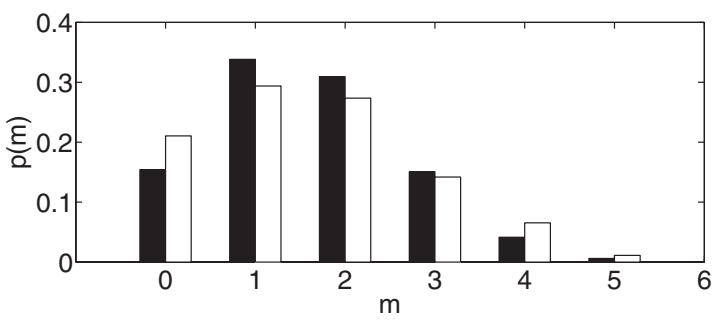

FIG. 6. The counting distributions of an expanded 1D checkerboard state, where every second site is occupied (black bars), and striped insulating pattern, where the first half of the sites is occupied and the remaining sites are empty (white bars), are clearly distinguishable. Parameters used: $z_{0}=1 \mathrm{~cm} ; \Delta=0.1 \mathrm{~cm} ; \Delta_{z}=$ $0.02 \mathrm{~mm} ; \kappa=1, N=12$.

on-site correlations. From Fig. 1, we see that this implies that the limit of small detectors has to be considered. However, if the detector is very small, all the terms are equal and the patterns are not distinguishable. One should thus consider an intermediate detector size.

In Fig. 6, we illustrate the effect for a 1D system of $N=12$ particles. We compare the counting distributions of a checkerboard-like state, where every second site is occupied, and a state where a block of six sites is occupied and a block of six sites is empty. In order to distinguish the two states, from Fig. 1, we choose a detector size of $\Delta=0.02 \mathrm{~mm}$ such that the ratio of the cross-correlation terms between neighboring sites and the autocorrelations is 0.6 . Figure 6 shows that the different occupation patterns are reflected in the counting distribution.

\section{Counting distribution of supersolid states}

In the weakly interacting regime, and in the case of long-range interactions, supersolid states with inhomogeneous occupation patterns $\alpha_{i}$ in Eq. (18) appear [37,38]. As for the states with different occupation patterns in the insulating regime, the characterization of supersolid states requires the limit where the cross-correlation terms for neighboring sites are comparable to the autocorrelation terms. We consider a supersolid state with $N$ sites and mean density $\alpha_{2 i}=\beta$ and $\alpha_{2 i-1}=\gamma$. As discussed in Sec. III B, the intensity of particles that is registered at the detector contains autocorrelations and cross-correlation terms. Depending on the detector position and size, the first $d_{\max }$ leading cross correlations have to be considered. For the limit where the cross-correlation terms for neighboring sites are the only non-negligible interference terms, the counting distribution Eq. (21) is given by a Poissonian distribution with mean

$$
\bar{m}=\frac{N}{2} A_{D}\left(\beta^{2}+\gamma^{2}\right)+2 N \operatorname{Re}\left(A_{N N}\right) \beta \gamma,
$$

where $A_{D}$ denotes the diagonal elements corresponding to the on-site correlations and $A_{N N}$ denotes the nearest-neighbor cross-correlation terms. Let us compare this to a superfluid state with a homogeneous density per site, $\left|\alpha_{i}\right|^{2}=\frac{|\beta|^{2}+\left|\gamma^{2}\right|}{2}$, for all $i$. The counting distribution Eq. (21) is thus given by a Poissonian distribution with mean

$$
\bar{m}=\frac{N}{2} A_{D}\left(\beta^{2}+\gamma^{2}\right)+N \operatorname{Re}\left(A_{N N}\right)\left(\beta^{2}+\gamma^{2}\right) .
$$

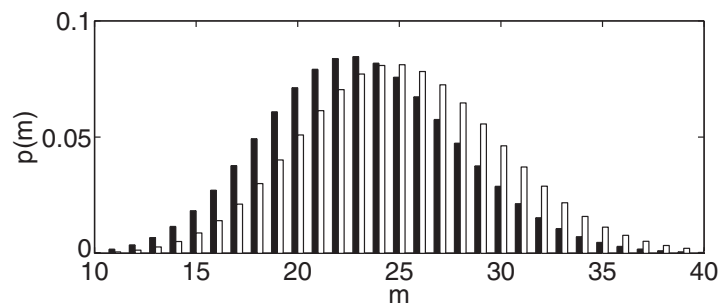

FIG. 7. The counting distributions of an expanded 1D supersolid state with $|\beta|^{2}=0.5$ and $|\gamma|^{2}=1.5$ (black bars) and a superfluid state $|\alpha|^{2}=1$ (white bars) are clearly distinguishable. Parameters used: $z_{0}=1 \mathrm{~cm} ; \Delta=1 \mathrm{~cm} ; \Delta_{z}=0.02 \mathrm{~mm} ; \kappa=1$, $N=40$.

From Eqs. (32) and (33) it is clear that a supersolid state can be distinguished from a superfluid state by particle counting when comparing the mean of the counting distributions of the two different states (Fig. 7). The mean of the counting distribution decreases as the supersolid structure appears in the state.

\section{Characterization of phase patterns}

The creation of artificial gauge fields in neutral atoms to mimic the dynamics of a charged particle in a magnetic field has attracted much attention [11]. Recently, magnetic Néel order has been simulated dressing an optical lattice with a fast oscillation [13] such that states of the form

$$
|\psi\rangle=\prod_{i}\left|\alpha_{i} e^{i \phi_{i}}\right\rangle
$$

can be created. The rapid oscillatory potential results in an effective tunneling rate between lattice sites which for certain values of the parameters can be tuned to negative values [12]. When the effective tunneling rate is negative, the energy is minimized when the phase between neighboring sites alternates. In this section, we show that the counting statistics allow for the characterization of the magnetic local phases in the states of Eq. (34). For illustration, let us consider possible phase patterns of Néel SF states appearing in square and triangular lattices [13]. The number distribution of a state described in Eq. (34) is Poissonian with mean value

$$
\bar{m}=N \alpha^{2} A_{D}+\alpha^{2} \sum_{i, j} A_{i, j} \cos \left(\phi_{i}-\phi_{j}\right),
$$

where $j=i+d$ and we take into account the leading correlations up to a term $d_{\max }$. It is clear from this expression that phase patterns will dramatically affect the number distribution. For the square lattice, we consider a phase difference of $\phi_{i}-$ $\phi_{i+1}= \pm \pi$ between neighboring sites. The sign of the tunneling is clearly reflected in the counting distribution (Fig. 8), as the mean decreases for states with phase patterns. For the triangular lattice, we consider a phase difference between neighboring sites of $\phi_{i}-\phi_{i+1}= \pm\{-2 \pi / 3,2 \pi / 3,4 \pi / 3\}$. As in the case of the square lattice, the change of the sign is clearly visible in the counting distribution (Fig. 9).

\section{SUMMARY}

We have studied the counting distributions of atoms falling from an optical lattice and propagating in the gravitational 


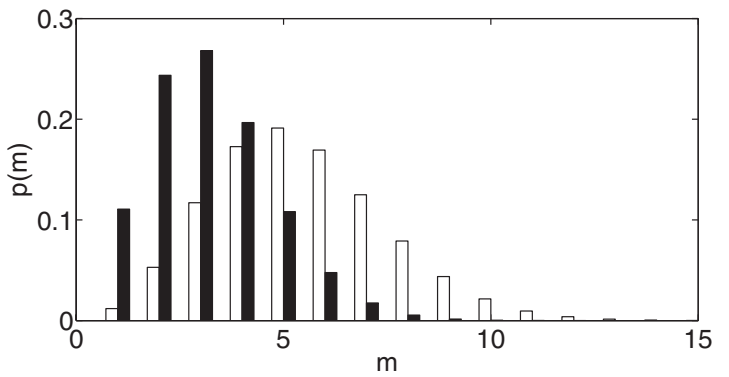

FIG. 8. The counting distribution of the particles released from a 2D square lattice in the $x y$ plane with positive tunneling rate (black bars) and negative tunneling rate (white bars). As the sign in the tunneling rate changes, the mean of the counting distribution decreases significantly. Parameters used: $\Delta_{x}=\Delta_{y}=$ $1 \mathrm{~cm} ; \Delta_{z}=0.1 \mathrm{~mm} ; z_{0}=1 \mathrm{~cm} ; a_{0}=415 \mathrm{~nm} ; N=100 ; d_{\max }=5$.

field. The atom number distribution gives access to high-order correlation functions and can thus be used to characterize the spatial correlations of the initial states. The intensity of atoms recorded at a detector located far from an optical lattice can be decomposed into autocorrelation and cross correlations between the expanding modes. The ratio between these terms depends crucially on the geometry of the detector.

In the limit when the detector is large compared to the expanded modes, the cross-correlation terms are negligible and only autocorrelations between the expanded modes are recorded in the detector. In this case only long-range correlations in the initially trapped states can be distinguished. In this situation, an SF state has a Poissonian number distribution while the counting distribution for an MI is sub-Poissonian. These two phases can also be readily distinguished from the joint probability distribution of counting the particles at two detectors. In the SF regime, the joint probability distribution is a product of the two independent number distributions while in the MI regime, the distributions are highly correlated. Also, the antibunching of bosons in the insulating regime is reflected in the correlation of the joint probability distribution. All these results are valid for SF and MI states irrespective of the occupation patterns.

When the detector is small compared to the expanded wave function, autocorrelations and cross correlations are recorded in the detector. We have shown that by choosing the size of the

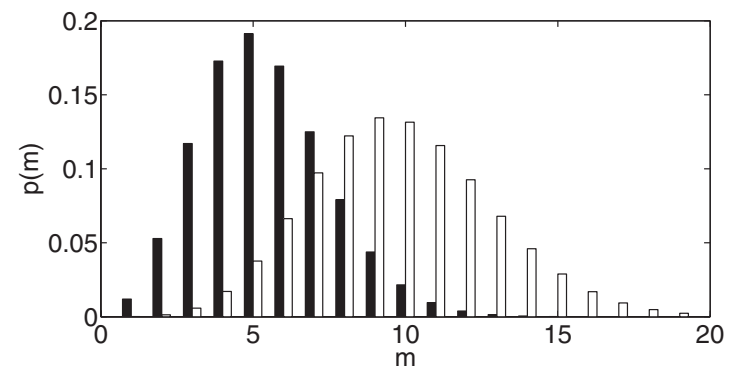

FIG. 9. The counting distribution of the particles released from a 2D triangular lattice in the $x y$ plane with positive tunneling rate (black bars) and negative tunneling rate (white bars). As the sign in the tunneling rate changes, the mean of the counting distribution decreases significantly. Parameters used: $\Delta_{x}=\Delta_{y}=1 \mathrm{~cm} ; \Delta_{z}=$ $0.1 \mathrm{~mm} ; z_{0}=1 \mathrm{~cm} ; a_{0}=415 \mathrm{~nm} ; N=100 ; d_{\max }=5$.

detector in an appropriate way, these contributions can be of the same order. In this case, different occupation patterns can be distinguished by particle counting after expansion both in the insulating as well as in the superfluid regime. Additionally, phase patterns in the SF regime are reflected in the number probability distribution. All the cases considered here show that superfluid phases with local phase or occupation patterns yield a Poissonian distribution with smaller mean compared to a homogeneous SF. In summary, we have shown that by choosing the detector size or, equivalently, the position of the detector with respect to the trapping lattice, one can characterize both the short- and long-range spatial correlations in the initial states.

\section{ACKNOWLEDGMENTS}

We acknowledge financial support from the Spanish MICINN project FIS2008-00784 (TOQATA), FIS201018799, Consolider Ingenio 2010 QOIT, EU-IP Project AQUTE, EU STREP project NAMEQUAM, ERC Advanced Grant QUAGATUA, the Ministry of Education of the Generalitat de Catalunya, and the Humboldt Foundation. M.R. is grateful to the MICINN of Spain for a Ramón y Cajal grant, and M.L. acknowledges support from the Alexander von Humboldt Foundation and the grant from the Hamburg Excellence Cluster "Frontiers in Quantum Photon Science."
[1] M. Lewenstein, A. Sanpera, V. Ahufinger, B. Damski, A. Sen(De), and U. Sen, Adv. Phys. 56, 243 (2007).

[2] T. Lahaye, C. Menotti, L. Santos, M. Lewenstein, and T. Pfau, Rep. Prog. Phys. 72, 126401 (2009).

[3] G. G. Batrouni and R. T. Scalettar, Phys. Rev. Lett. 84, 1599 (2000); P. Sengupta, L. P. Pryadko, F. Alet, M. Troyer, and G. Schmid, ibid. 94, 207202 (2005); D. L. Kovrizhin, G. V. Pai, and S. Sinha, Europhys. Lett. 72, 162 (2005); K. Góral, L. Santos, and M. Lewenstein, Phys. Rev. Lett. 88, 170406 (2002).

[4] C. Menotti, C. Trefzger, and M. Lewenstein, Phys. Rev. Lett. 98, 235301 (2007).
[5] C. Trefzger, C. Menotti, and M. Lewenstein, Phys. Rev. A 78, 043604 (2008).

[6] B. Capogrosso-Sansone, C. Trefzger, M. Lewenstein, P. Zoller, and G. Pupillo, Phys. Rev. Lett. 104, 125301 (2010).

[7] L. Pollet, J. D. Picon, H. P. Büchler, and M. Troyer, Phys. Rev. Lett. 104, 125302 (2010).

[8] U. Dorner, P. Fedichev, D. Jaksch, M. Lewenstein, and P. Zoller, Phys. Rev. Lett. 91, 073601 (2003).

[9] M. Pons, V. Ahufinger, C. Wunderlich, A. Sanpera, S. Braungardt, A. Sen(De), U. Sen, and M. Lewenstein, Phys. Rev. Lett. 98, 023003 (2007); S. Braungardt, A. Sen(De), U. Sen, and M. Lewenstein, Phys. Rev. A 76, 042307 (2007). 
[10] K. Baumann, C. Guerlin, F. Brennecke, and T. Esslinger, Nature 464, 1301 (2010).

[11] J. Dalibard, F. Gerbier, G. Juzeliūnas, and P. Öhberg, e-print arXiv:1008.5378 (2010).

[12] A. Eckardt, C. Weiss, and M. Holthaus, Phys. Rev. Lett. 95, 260404 (2005); H. Lignier, C. Sias, D. Ciampini, Y. Singh, A. Zenesini, O. Morsch, and E. Arimondo, ibid. 99, 220403 (2007).

[13] A. Eckardt, P. Hauke, P. Soltan-Panahi, C. Becker, K. Sengstock, and M. Lewenstein, Eur. Phys. Lett. 89, 10010 (2010); J. Struck, C. Ölschläger, R. Le Targat, P. Soltan-Panahi, A. Eckardt, M. Lewenstein, P. Windpassinger, and K. Sengstock, Science 333, 996 (2011).

[14] E. Altman, E. Demler, and M. D. Lukin, Phys. Rev. A 70, 013603 (2004).

[15] K. Eckert, O. Romero-Isart, M. Rodríguez, M. Lewenstein, E. Polzik, and A. Sanpera, Nat. Phys. 4, 50 (2008); D. Porras and J. I. Cirac, Phys. Rev. A 78, 053816 (2008).

[16] J. Grondalski, P. M. Alsingy, and I. H. Deutsch, Opt. Express 5, 11 (1999).

[17] I. B. Mekhov, C. Maschler, and H. Ritsch, Nat. Phys. 3, 319 (2007); Phys. Rev. Lett. 98, 100402 (2007); W. Chen, D. Meiser, and P. Meystre, Phys. Rev. A 75, 023812 (2007); L. Guo, S. Chen, B. Frigan, L. You, and Y. Zhang, ibid. 79, 013630 (2009).

[18] S. Braungardt, A. Sen(De), U. Sen, R. J. Glauber, and M. Lewenstein, Phys. Rev. A 78, 063613 (2008).

[19] R. W. Cherng and E. Demler, New J. Phys. 9, 7 (2007).

[20] W. Belzig, C. Schroll, and C. Bruder, Phys. Rev. A 75, 063611 (2007).

[21] S. Braungardt, M. Rodríguez, A. Sen(De), U. Sen, R. J. Glauber, and M. Lewenstein, Phys. Rev. A 83, 013601 (2011).

[22] T. C. Liebisch, A. Reinhard, P. R. Berman, and G. Raithel, Phys. Rev. Lett. 95, 253002 (2005); M. Viteau, M. G. Bason,
J. Radogostowicz, N. Malossi, D. Ciampini, O. Morsch, and E. Arimondo, e-print arxiv:1103.4232 (2011).

[23] T. Gericke, P. Würtz, D. Reitz, T. Langen, and H. Ott, Nat. Phys. 4, 949 (2008).

[24] N. Gemelke, X. Zhang, C.-L. Hung, and C. Chin, Nature 460, 995 (2009).

[25] W. S. Bakr, A. Peng, M. E. Tai, R. Ma, J. Simon, J. I. Gillen, S. Fölling, L. Pollet, and M. Greiner, Science 329, 547 (2010).

[26] J. F. Sherson, C. Weitenberg, M. Endres, M. Cheneau, I. Bloch, and S. Kuhr, Nature 467, 68 (2010).

[27] T. Stöferle, H. Moritz, C. Schori, M. Köhl, and T. Esslinger, Phys. Rev. Lett. 92, 130403 (2004).

[28] M. Schellekens, R. Hoppeler, A. Perrin, J. Viana Gomes, D. Boiron, A. Aspect, and C. I. Westbrook, Science 310, 648 (2005).

[29] T. Jeltes et al., Nature 445, 402 (2007).

[30] D. Jaksch, C. Bruder, J. I. Cirac, C. W. Gardiner, and P. Zoller, Phys. Rev. Lett. 81, 3108 (1998); M. Greiner, O. Mandel, T. Esslinger, T. W. Hänsch, and I. Bloch, Nature 415, 39 (2010).

[31] T. Kramer, C. Bracher, and M. Kleber, J. Phys. A 35, 8361 (2002).

[32] R. J. Glauber, in Quantum Optics and Electronics, edited by B. DeWitt, C. Blandin, and C. Cohen-Tannoudji (Gordon \& Breach, New York, 1965), pp. 63-185.

[33] K. E. Cahill and R. J. Glauber, Phys. Rev. A 59, 1538 (1999).

[34] J. Grochmalicki and M. Lewenstein, Phys. Rep. 208, 189 (1991).

[35] F. T. Arecchi, A. Berné, and A. Sona, Phys. Rev. Lett. 17, 260 (1966).

[36] W. Krauth, M. Caffarel, and J.-P. Bouchaud, Phys. Rev. B 45, 3137 (1992); K. Sheshadri, H. R. Krishnamurthy, R. Pandit, and T. V. Ramakrishnan, Europhys. Lett. 22, 257 (1993).

[37] A. J. Leggett, Phys. Rev. Lett. 25, 1543 (1970).

[38] N. Prokof'ev, Adv. Phys. 56, 381 (2007). 